\title{
Low-dose metronomic doxorubicin inhibits mobilization and differentiation of endothelial progenitor cells through REDD1-mediated VEGFR-2 downregulation
}

\author{
Minsik Park ${ }^{1, \#}$, Ji Yoon Kim ${ }^{2, \#}$, Joohwan Kim ${ }^{1, \#}$, Jeong-Hyung Lee ${ }^{3}$, Young-Guen Kwon ${ }^{4}$ E Young-Myeong Kim ${ }^{1, *}$ \\ ${ }^{1}$ Department of Molecular and Cellular Biochemistry, Kangwon National University School of Medicine, Chuncheon 24341, ${ }^{2}$ Department of \\ Anaesthesiology and Pain Medicine, Hanyang University Hospital, Seoul 04763, ${ }^{3}$ Department of Biochemistry, Kangwon National University, \\ Chuncheon 24341, ${ }^{4}$ Department of Biochemistry, College of Life Science and Biotechnology, Yonsei University, Seoul 03722, Korea
}

Low-dose metronomic chemotherapy has been introduced as a less toxic and effective strategy to inhibit tumor angiogenesis, but its anti-angiogenic mechanism on endothelial progenitor cells (EPCs) has not been fully elucidated. Here, we investigated the functional role of regulated in development and DNA damage response 1 (REDD1), an endogenous inhibitor of mTORC1, in low-dose doxorubicin (DOX)-mediated dysregulation of EPC functions. DOX treatment induced REDD1 expression in bone marrow mononuclear cells (BMMNCs) and subsequently reduced mTORC1-dependent translation of endothelial growth factor (VEGF) receptor (Vegfr)-2 mRNA, but not that of the mRNA transcripts for Vegfr-1, epidermal growth factor receptor, and insulin-like growth factor-1 receptor. This selective event was a risk factor for the inhibition of BMMNC differentiation into EPCs and their angiogenic responses to VEGF-A, but was not observed in Redd1-deficient BMMNCs. Low-dose metronomic DOX treatment reduced the mobilization of circulating EPCs in B16 melanoma-bearing wild-type but not Redd1-deficient mice. However, REDD1 overexpression inhibited the differentiation and mobilization of EPCs in both wild-type and Redd1deficient mice. These data suggest that REDD1 is crucial for metronomic DOX-mediated EPC dysfunction through the translational repression of Vegfr-2 transcript, providing REDD1 as a potential therapeutic target for the inhibition of tumor angiogenesis and tumor progression. [BMB Reports 2021; 54(9): 470-475]

*Corresponding author. Tel: +82-33-250-8831; Fax: +82-33-259-5748; E-mail: ymkim@kangwon.ac.kr

${ }^{\#}$ These authors contributed equally to this work.

https://doi.org/10.5483/BMBRep.2021.54.9.096

Received 19 July 2021, Revised 29 July 2021, Accepted 10 August 2021

Keywords: Angiogenesis, EPCs, Metronomic chemotherapy, REDD1, VEGFR-2

\section{INTRODUCTION}

Tumor blood vessels are formed by two different mechanisms, namely tumor angiogenesis and vasculogenesis, mostly through the vascular endothelial growth factor (VEGF)/VEGF receptor-2 (VEGFR-2) axis (1). Tumor angiogenesis occurs through the proliferation and migration of endothelial cells from pre-existing neighboring blood vessels, whereas tumor vasculogenesis is initiated by recruiting bone marrow-derived VEGFR- ${ }^{+}$endothelial progenitor cells (EPCs), which differentiate into mature endothelial cells (2). Thus, VEGF released from the tumor microenvironment plays an important role in the formation of tumor blood vessels, leading to tumor progression and metastasis. It is now well understood that blocking the VEGF/VEGFR-2 pathway can act as a therapeutic strategy for cancer patients by inhibiting the functions of endothelial cells and EPCs $(3,4)$.

Low-dose metronomic chemotherapy (LDMC) has recently been attracting attention as a promising alternative to conventional maximum-tolerated dose chemotherapy (5). This regimen is thought to effectively target both activated or proliferating tumor endothelial cells as well as EPCs, which are more sensitive to low-dose anticancer drugs than normal endothelial and tumor cells $(3,4)$. Indeed, LDMC has been shown to effectively inhibit the tumor progression by suppressing tumor angiogenesis through functional impairment of tumor endothelial cells and bone marrow mononuclear cell (BMMNC)-derived EPCs $(4,5)$. This is affected by a systemic increase in anti-angiogenic factors, including thrombospondin-1 (TSP-1), as well as a reduction in pro-angiogenic factors, such as VEGF and VEGFR-2 $(3,6)$. However, the molecular mechanism underlying anti-angiogenesis by LDMC has not been clearly elucidated.

The protein regulated in development and DNA damage response 1 (REDD1) is upregulated upon the treatment with chemotherapeutic drugs, including doxorubicin (DOX) $(7,8)$. REDD1 functions as an endogenous mTORC1 inhibitor as well as an atypical NF-kB activator $(9,10)$. The mTORC1 pathway plays a crucial role in the regulation of various cellular functions, such as protein biosynthesis and angiogenesis (11). Indeed, the mTOR inhibitor rapamycin suppresses tumor angiogenesis by

ISSN: 1976-670X (electronic edition)

Copyright (c) 2021 by the The Korean Society for Biochemistry and Molecular Biology

(c) This is an open-access article distributed under the terms of the Creative Commons Attribution Non-Commercial License (http://creativecommons.org/licenses/by-nc/4.0) which permits unrestricted non-commercial use, distribution, and reproduction in any medium, provided the original work is properly cited. 
downregulating VEGF/VEGFR expression through the inhibition of translational initiation $(12,13)$. We have recently reported that LDMC-induced REDD1 suppresses tumor angiogenesis and tumor progression via functional impairment of tumor endothelial cells through translational repression of Vegfr-2 mRNA by inhibiting the mTORC1 pathway (6). However, there is no evidence that REDD1 inhibits the differentiation and function of EPCs.

Here, we hypothesized that REDD1 is responsible for the LDMC-mediated impairment of EPC function and tumor angiogenesis. We found that LDMC with DOX increased REDD1 expression, repressed Vegfr-2 mRNA translation, and decreased the mobilization and differentiation of EPCs in wild-type (WT) but not Redd $1^{-1-}$ mice. Moreover, REDD1 overexpression inhibited EPC mobilization and differentiation in both mouse types. These findings suggest that REDD1 is crucial for low-dose DOX-mediated impairment of EPC function by translational repression of VEGFR-2 expression.

\section{RESULTS}

\section{Low-dose DOX induces REDD1 and downregulates VEGFR-2 in BMMNC-derived EPCs}

Since LDMC induces REDD1 expression and impairs endothelial cell functions (3, 6-8), we examined whether low-dose DOX treatment-induced REDD1 regulates angiogenic receptors in BMMNC-derived EPCs. When treated with non-cytotoxic doses (1-3 $\mathrm{nM}$ ) of DOX as determined by assaying cytotoxicity and cell viability (Supplementary Fig. 1A, B), BMMNC-derived EPCs showed dose-dependently increased REDD1 expression, along with downregulation of VEGFR-2; however, there were no differences in the expression levels of VEGFR-1, epithermal growth factor receptor (EGFR), and insulin growth factor-1 receptor $\beta$ (IGF-1R $\beta$ ) (Fig. 1A). Since REDD1 is known as an endogenous mTORC1 inhibitor (9), we next compared the effects of DOX, REDD1, and the mTORC1 inhibitor rapamycin on VEGFR-2 expression. Treatment with DOX or rapamycin or adenoviral overexpression of REDD1 suppressed VEGFR-2 expression in BMMNC-derived EPCs, without affecting its mRNA levels, compared with untreated control cells (Fig. 1B, C), suggesting that DOX selectively inhibits the translational expression of VEGFR-2 by REDD1-dependent mTORC1 inhibition. We further examined the possible role of REDD1 in DOX-mediated VEGFR-2 downregulation by analyzing the mTORC1 signaling pathway and polysome profiling. DOX treatment or REDD1 overexpression inhibited the phosphorylation of mTOR, p70 ribosomal S6 kinase (S6K), and eukaryotic translational initiation factor 4E-binding protein 1 (4E-BP1) (Fig. 1D). Consistent with this, both treatments decreased the assembly of high-molecularweight polysome complexes and polysome-associated Vegfr-2 mRNA levels compared with control cells, without affecting the polysomal mRNA levels of Vegfr-1, Egfr, and Igf-1r (Fig. 1E, $\mathrm{F}$ and Supplementary Fig. 1C). Collectively, these results sug-
A

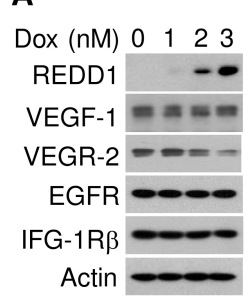

B

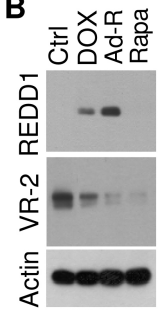

D
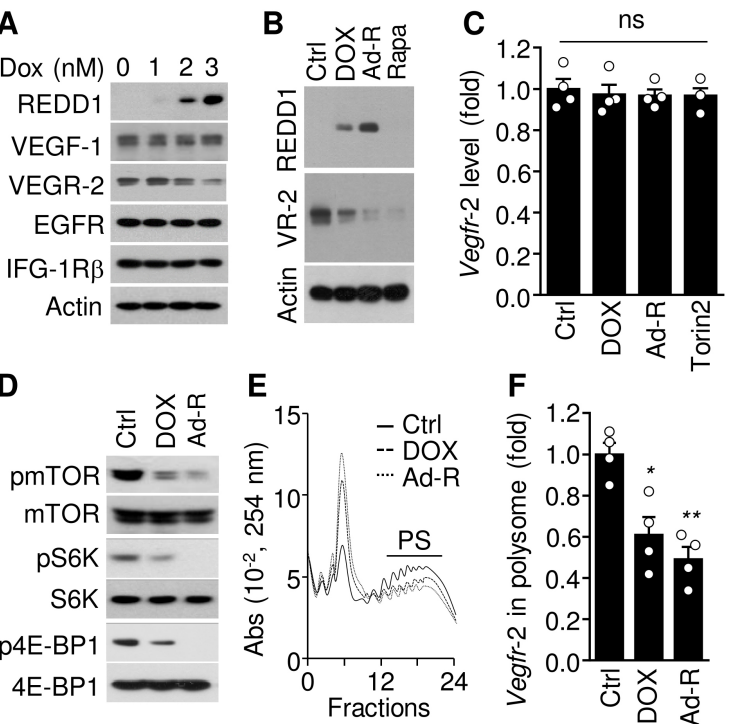

Fig. 1. Low-dose DOX treatment-induced REDD1 downregulates VEGFR-2 in BMMNC-derived EPCs. BMMNCs were cultured in EGM-2MV medium for 4 days and treated with DOX (at the indicated concentrations or $3 \mathrm{nM}$ ) or rapamycin (Rapa, $20 \mathrm{nM}$ ) or infected with Ad-Redd1 (Ad-R, $400 \mathrm{MOI}$ ) for $24 \mathrm{~h}$. (A, B) Expression levels of the target proteins were determined by western blot analysis. (C) Levels of Vegfr-2 mRNA were determined by qRT-PCR analysis $(n=4)$. (D) Levels of phosphorylated mTOR, S6K, and 4E-BP-1 were analyzed by western blot analysis. (E) Representative polysome profiling was analyzed by sucrose density gradient ultracentrifugation. PS, polysomes. (F) Levels of Vegfr-2 mRNA associated with high-molecular-weight polysomes were determined by qRT-PCR analysis $(n=4)$. ${ }^{*} \mathrm{P}<0.05 ; * * P<$ 0.01 ; ns, not significant vs. control.

gest that DOX-induced REDD1 negatively regulates VEGFR-2 expression via mTOR-dependent repression of its mRNA translation.

\section{DOX inhibits the differentiation of WT but not Redd1 ${ }^{-1-}$ BMMNCs into EPCs}

Since VEGFR-2 plays a crucial role in EPC mobilization and differentiation $(14,15)$, we examined the effects of DOX treatment and REDD1 overexpression on EPC differentiation from cultured BMMNCs isolated from WT and Redd $1^{-1-}$ mice. DOX treatment significantly inhibited the in vitro differentiation of WT but not Redd $1^{-1-}$ BMMNCs into EPCs, as determined by counting double-positive cells stained with Dil-ac-LDL and FITC-UEA-1 and quantifying their fluorescence intensity, a characteristic feature of EPCs (Fig. 2A and Supplementary Fig 2A). However, overexpression of REDD1 effectively suppressed double staining with Dil-ac-LDL and FITC-UEA-1 in both WT and Redd $1^{-1-}$ BMMNC-derived EPCs (Fig. 2A and Supplementary Fig. 2A). Under the same experimental conditions, we further analyzed the expression of the endothelial cell markers VEGFR-2 and CD31 by flow cytometry. DOX treatment signi- 
A
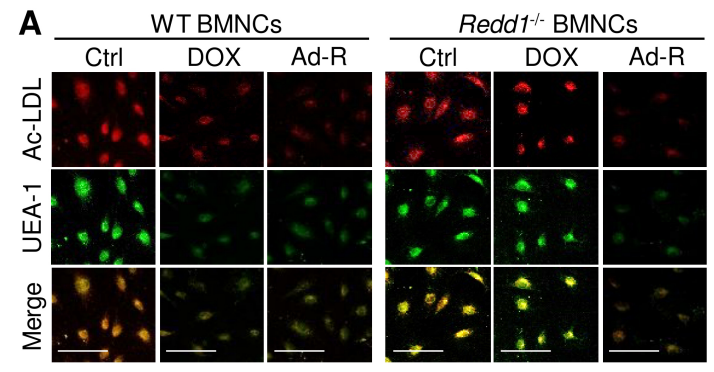

B

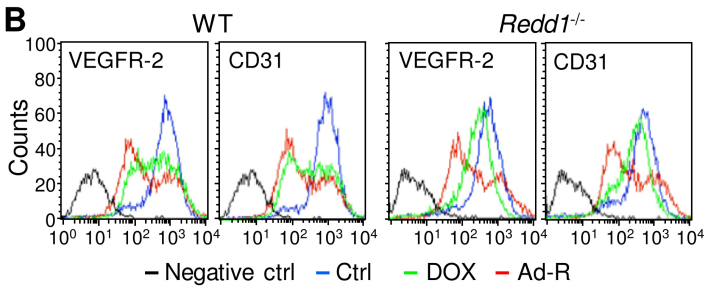

Fig. 2. DOX inhibits differentiation of WT but not Redd $1^{-1-}$ BMMNCs into EPCs. BMMNCs isolated from WT and Redd $1^{-1-}$ mice were treated with DOX or infected with Ad-Redd1 (Ad-R) for 24 h. (A) EPCs were identified by confocal microscopy after staining with Dil-ac-LDL and FITC-UEA-1. Bar, $100 \mu \mathrm{m}$. (B) Cells were labelled with antibodies for VEGFR-2 and CD31 and analyzed by flow cytometry.

ficantly decreased the expression levels of VEGFR-2 and CD31 in BMMNC-derived EPCs obtained from WT but not Redd $1^{-1-}$ mice; however, REDD1 overexpression suppressed the expression of the endothelial cell markers in both $\mathrm{WT}$ and Redd $1^{-1-}$ BMMNC-derived EPCs (Fig. 2 and Supplementary Fig. 2B, C). These results suggest that DOX-induced REDD1 negatively regulates the differentiation of BMMNCs into EPCs via downregulation of VEGFR-2.

\section{DOX inhibits the angiogenic activity of WT but not Redd $^{-1-}$ BMMNC-derived EPCs}

Since the VEGF/VEGFR-2 system regulates the endothelial nitric oxide synthase (eNOS)/NO axis, which is another functional marker of EPCs (16), we investigated whether low-dose DOX regulates eNOS-dependent $\mathrm{NO}$ production in BMMNC-derived EPCs. VEGF-A stimulation significantly increased NO production in EPCs normally differentiated from both WT and Redd $1^{-1-}$ BMMNCs; however, VEGF-A-responsive NO production was suppressed in EPCs differentiated from DOX-treated WT but not Redd $1^{-1-}$ BMMNCs (Fig. 3A and Supplementary Fig. 3A). In contrast, VEGF-A-induced NO production was inhibited in EPCs differentiated from both REDD1-overexpressed WT and Redd $^{-1-}$ BMMNCs (Fig. 3A and Supplementary Fig. 3A). This suggests that DOX-induced REDD1 downregulates VEGF-A-mediated NO production in BMMNC-derived EPCs via VRGFR-2 downregulation. Next, we examined the effects of DOX on the in vitro angiogenic activity of BMMNC-derived EPCs. Consistent with the results observed for NO production, EPCs differ-

\section{A}

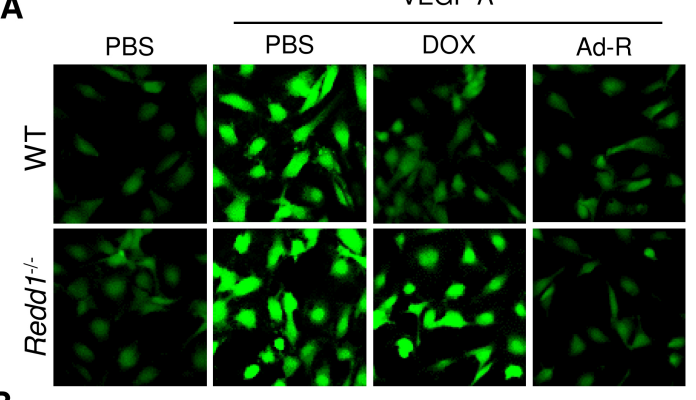

B

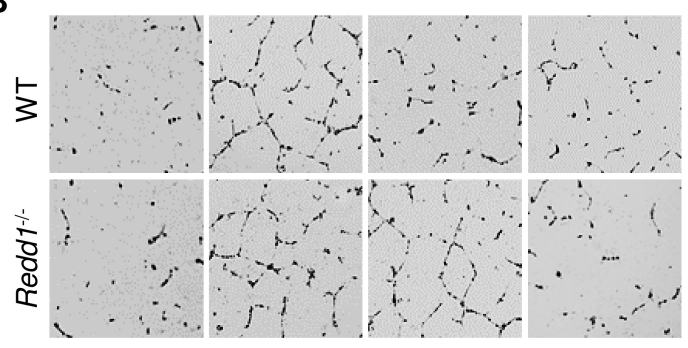

Fig. 3. DOX inhibits NO production and angiogenic activity of WT but not Redd $1^{-1-}$ BMMNC-derived EPCs. (A) BMMNCs isolated from WT and Redd1-/- mice were treated with DOX or infected with Ad-Redd1 (Ad-R), followed by measurement of VEGF-A-induced intracellular NO production using a DAF-FM (a NO-sensitive probe)-based confocal microscopic analysis. (B) Tube formation of BMMNC-derived EPCs in response to VEGF-A was imaged using an inverted microscope.

entiated from DOX-treated WT but not Redd $1^{-1-}$ BMMNCs showed a significant decrease in VEGF-A-induced angiogenic properties, such as tube formation and migration, compared with untreated control cells; however, EPCs differentiated from both REDD1-overexpressed WT and Redd $1^{-1-}$ BMMNCs showed reduced angiogenic responses to VEGF-A (Fig. 3B and Supplementary Fig. 3B, C). Collectively, these results suggest that DOX-induced REDD1 plays an important role in impairing the angiogenic activity of BMMNC-derived EPCs by downregulating VEGFR-2.

\section{Metronomic DOX decreases EPC mobilization in tumor-bearing WT but not Redd1 ${ }^{-1-}$ mice}

Since LDMC inhibits tumor angiogenesis by impairing the mobilization and recruitment of EPCs as well as pre-existing endothelial cell functions $(3,17,18)$, we investigated whether metronomic DOX treatment regulates the mobilization of EPCs from the bone marrow into the peripheral blood in B16 melanomabearing WT and $R e d d 1^{-1-}$ mice as an in vivo model for DOXmediated inhibition of tumor angiogenesis (8). Metronomic DOX treatment markedly decreased the number of circulating EPCs expressing CD $34^{+}$NEGFR-2 ${ }^{+}$in B16 melanoma-bearing WT but not Redd $1^{-1-}$ mice; however, intravenous injection of Ad-Redd1 potentially reduced the circulating EPC population in both tumor-bearing WT and Redd $1^{-1-}$ mice (Fig. 4). These 
A

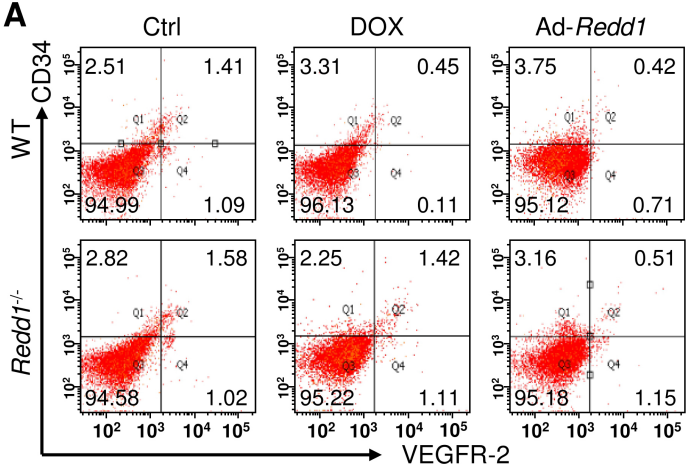

B

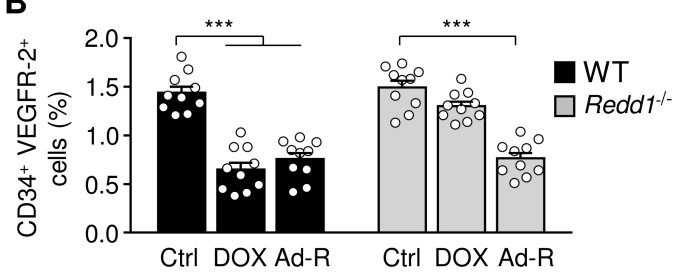

Fig. 4. Metronomic DOX decreases EPC mobilization in tumor-bearing WT but not Redd $1^{-1-}$ mice. B16 melanoma-bearing WT and Redd $1^{-l-}$ mice were metronomically treated with DOX or intravenously injected with Ad-Redd1. (A) Representative dot plots of VEGFR-2 ${ }^{+}$/ $\mathrm{CD}_{3}{ }^{+}$EPCs were obtained in peripheral blood of mice using flow cytometry. (B) The number of VEGFR- $2^{+} / \mathrm{CD} 34^{+}$EPCs was quantified by flow cytometric analysis ( $\mathrm{n}=10$ mice/group). ${ }^{* * *} \mathrm{P}<0.001$; ns, not significant vs. control.

results suggest that REDD1 is a crucial mediator of metronomic DOX-induced suppression of EPC mobilization from the bone marrow in tumor-bearing mice.

\section{DISCUSSION}

We investigated the functional role and molecular mechanism of REDD1, a cellular mTORC1 inhibitor, in the inhibitory effects of low-dose DOX treatment on the differentiation and mobilization of EPCs. Our data showed that low-dose DOX treatment inhibited the differentiation of BMMNCs into EPCs by upregulating REDD1 and subsequently repressing mTORC1dependent Vegfr-2 mRNA translation, thus leading to the inhibition of VEGF-A-induced NO production and angiogenic activity of WT but not Redd $1^{-1-}$ BMMNC-derived EPCs. Furthermore, low-dose metronomic DOX treatment decreased the number of circulating EPCs in B16 melanoma-bearing WT mice but not Redd $1^{-1-}$ mice. Notably, adenoviral overexpression of REDD1 inhibited the in vitro differentiation of both WT and Redd $1^{-1-}$ BMMNCs into EPCs and also suppressed their mobilization from bone marrow in both tumor-bearing WT and Redd $1^{-1-}$ mice. Therefore, we have concluded that metronomic DOX treatment could impair EPC function and tumor angiogenesis through REDD1-dependent translational repression of Vegfr-2 mRNA.

Although angiogenic processes are well understood, it has been shown that several single antiangiogenic therapies targeting tumor endothelial cells do not always effectively inhibit tumor angiogenesis and tumor progression (19), suggesting that tumor angiogenesis is not regulated only by the stimulation of preexisting endothelial cells. Asahara and his colleagues have identified a population of circulating EPCs with characteristics similar to those of embryonic angioblasts that contribute to angiogenesis in ischemic tissues and tumors $(2,20,21)$. In general, EPCs promote tumor angiogenesis in three consecutive steps, namely mobilization from bone marrow, recruitment to the tumor site, and differentiation into mature endothelial cells; all of these processes can be regulated by the signaling pathways induced by growth factors or chemokines and their corresponding receptors, including VEGF-AVVEGFR-2, stromal cell-derived factor-1 (SDF-1)/CXCR4, and basic fibroblast growth factor/its receptor (3). Therefore, treatment with blockades of the VEGF-A/VEGFR-2 and SDF-1/CXCL4 axes has been shown to decrease the number of circulating EPCs and their recruitment into tumor tissues in patients with rectal cancer and Lewis lung cancer mouse models, respectively, resulting in amelioration of tumor angiogenesis and tumor progression $(22,23)$. LDMC has been shown to reduce the number of circulating EPCs in tumor-bearing mice and cancer patients, leading to a parallel decrease in tumor angiogenesis and vessel density (3); however, the mechanism by which LDMC inhibits EPC-mediated tumor angiogenesis remains unclear. Our results demonstrate that low-dose DOX treatment downregulates VEGFR-2 expression along with the suppression of differentiation, mobilization, NO production, and angiogenic activity of EPCs in WT but not Redd $1^{-1-}$ mice, suggesting that REDD1 plays a crucial role in metronomic DOX-induced inhibition of angiogenic function of EPCs via VEGFR-2 downregulation.

REDD1 is required for the dissociation of TSC2/14-3-3 and subsequent inhibition of the mTORC1 pathway, which is essential for controlling cap-dependent mRNA translation $(9,24)$, and is induced by various stress conditions and chemotherapeutic drugs, including DOX $(3,7,9)$. Consistent with this, we found that DOX upregulated REDD1 and subsequently impeded the assembly of high-molecular-weight-polysome complexes by inhibiting the mTORC1-dependent phosphorylation of S6K and 4E-BP1. Notably, DOX treatment caused translational repression of Vegfr-2 mRNA, but not that of Vegfr-1, Igf-1r, and Egfr mRNAs, in WT BMMNC-derived EPCs; however, REDD1 overexpression effectively repressed the translation of Vegfr-2 transcript in both WT and Redd1 $1^{-1-}$ BMMNC-derived EPCs. These results suggest that DOX selectively represses Vegfr-2 translation via REDD1-dependent inhibition of the mTORC1 pathway. This selectivity may be associated with the structural differences in the $5^{\prime}$-untranslated regions (5'-UTRs) of those genes. Indeed, the REDD1-mTORC1 axis has been shown to inhibit cap-dependent translation of Vegfr-2 mRNA, which does not contain an internal ribosome entry site (IRES) within 
its $5^{\prime}$-UTR; however, there is no putative or authentic IRES within the 5 '-UTRs of Vegfr-1, Egfr, and Igf-1r transcripts (8, 25). Thus, DOX-induced REDD1 inhibits the mobilization and differentiation of EPCs by selectively repressing Vegfr-2 mRNA translation, as shown upon the treatment with the mTORC1 inhibitor rapamycin (26).

In conclusion, our results demonstrate that low-dose metronomic DOX treatment inhibited the differentiation and mobilization of EPCs from bone marrow through selective translational repression of Vegfr-2 mRNA by upregulating REDD1, ultimately resulting in the functional inhibition of EPCs. Thus, the REDD1-mTORC1-VEGFR-2 axis plays an important role in DOX-induced inhibition of EPC-mediated tumor angiogenesis and is considered to be a key pathway of metronomic DOXsensitive EPC dysfunction. These findings also provide evidence that REDD1 is a novel therapeutic target for controlling tumor angiogenesis by dysregulating EPC mobilization and differentiation.

\section{MATRIALS AND METHODS}

Additional materials and methods are available in the Supplemental Information.

\section{Cell culture and treatment}

Bone marrow was collected from the tibia and femur of 6-week-old male mice in a $50 \mathrm{ml}$ conical tube immediately after sacrifice by $\mathrm{CO}_{2}$ inhalation. BMMNCs were isolated by density gradient centrifugation using Histopaque-1083 (SigmaAldrich), suspended in EGM-2MV (Lonza, \#CC-3202), and seeded on gelatin-coated plates at a density of $1 \times 10^{6} \mathrm{cell} / \mathrm{s} / \mathrm{cm}^{2}$. Four days later, non-adherent cells were washed off with phosphate-buffered saline (PBS), cultured in fresh media for 2 days, and then treated daily with DOX ( $3 \mathrm{nM})$ or rapamycin $(20 \mathrm{nM})$ in fresh media for $24 \mathrm{~h}$. In addition, adherent BMMNCs were infected with adenovirus expressing mouse Redd1 (AdRedd1) and empty adenovirus (Ad-Control) (8) at a multiplicity of infection (MOI) of 400 for $4 \mathrm{~h}$ and cultured in fresh medium for $20 \mathrm{~h}$. BMMNCs were used to assess gene expression and differentiation.

\section{Polysome assay}

Cells were lysed in $400 \mu$ lysis buffer $[15 \mathrm{mM}$ Tris- $\mathrm{HCl}(\mathrm{pH}$ 7.4), $0.3 \mathrm{M} \mathrm{NaCl}, 15 \mathrm{mM} \mathrm{MgCl} 2,0.1 \mathrm{mg} / \mathrm{ml}$ cycloheximide, and $200 \mathrm{U} / \mathrm{ml}$ Superase- $\mathrm{In}^{\mathrm{TM}}$ (Ambion, Waltham, MA, USA)]. After centrifugation at $2000 \times \mathrm{g}$ for $5 \mathrm{~min}$ to remove nuclei and mitochondria, heparin (a broad-range RNase inhibitor) was added to the supernatant at a final concentration of 200 $\mu \mathrm{g} / \mathrm{ml}$. After removing debris by centrifugation at $10,000 \times \mathrm{g}$ for $5 \mathrm{~min}$, the cytosolic supernatants were overlaid on a 20-50\% sucrose gradient (total volume: $5 \mathrm{ml}$ ) and centrifuged at $39,000 \mathrm{rpm}$ for $120 \mathrm{~min}$ in a Beckman SW-55Ti rotor at $4^{\circ} \mathrm{C}$. After centrifugation, gradients were fractionated in 0.2 $\mathrm{ml}$-aliquots with continuous monitoring of absorbance at 254 $\mathrm{nm}$. The fractions containing high-molecular-weight polysomes were pooled.

\section{Differentiation of BMMNCs into EPCs}

BMMNCs treated with drugs or infected with Ad-Redd1 were incubated with $10 \mu \mathrm{g} / \mathrm{ml}$ of Dil-Ac-LDL (Invitrogen, \#L3483) in a $\mathrm{CO}_{2}$ incubator at $37^{\circ} \mathrm{C}$ for $2 \mathrm{~h}$. The cells were then washed twice, fixed in $2 \%$ paraformaldehyde, and incubated with 10 $\mu \mathrm{g} / \mathrm{ml}$ of FITC-UEA-1 (Sigma-Aldrich, \#L9006) for $2 \mathrm{~h}$ at room temperature. Images were obtained using a Zeiss LSM 880 laser scanning confocal microscope with Airyscan (Zeiss, Oberkochen, Germany). In addition, the cells were detached with a nonenzymatic cell dissociation solution (Sigma-Aldrich, \#C5789) to avoid the destruction of cell membrane markers. The cells were incubated with PE-conjugated anti-mouse VEGFR-2 antibody (Biolegend, \#138012), APC-conjugated anti-mouse PECAM-1 (CD31) antibody (Biolegend, \#102410), or isotype-matched IgG controls. The expression levels of the target proteins were determined using flow cytometry.

\section{Measurement of circulating EPCs in tumor-bearing mice}

Wild-type and $R e d d 1^{-1-}$ mice were fed with a standard chow diet and tap water ad libitum in a pathogen-free animal facility, as described previously (8). All animal experiments were performed in accordance with the Guide for the Care and Use of Laboratory Animals (National Institutes of Health, USA) and approved by the Animal Ethics Committee of Kangwon National University (approval number 2017-0260A). Murine melanoma B16F1 cells $\left(5 \times 10^{6}\right)$ suspended in $100 \mu \mathrm{l}$ saline were injected into the subcutaneous dorsa of the right flank of 6weeks-old male WT and Redd $1^{-1-}$ mice. After the detection of the tumors on day 5 , mice were treated with saline or DOX $(0.2 \mathrm{mg} / \mathrm{kg}$ every 2 days, intraperitoneally) or injected with Ad-Redd1 $\left(1 \times 10^{9}\right.$ plaque-forming units in a total volume of $50 \mu \mathrm{l}$, intravenously) on days 5 and 10 after tumor inoculation. On day 16 after tumor cell injection, mice were anesthetized using $2.5 \%(\mathrm{v} / \mathrm{v})$ Avertin, and whole blood was collected in tubes containing EDTA by cardiac puncture. Blood (200 $\mu \mathrm{l})$ was incubated for $15 \mathrm{~min}$ at room temperature and in darkness with ACP-conjugated anti-mouse CD34 antibody (Biolegend, \#128612), PE-conjugated anti-mouse VEGFR-2 antibody (Biolegend, \#121906), or isotype-matched IgG controls. Erythrocytes were lysed using FACS lysing solution (Biolegend, \#420302) and washed with PBS containing $0.5 \%$ bovine serum albumin. All samples were analyzed by flow cytometry.

\section{Statistical analysis}

Statistical analyses were performed using GraphPad Prism software (version 6.07). All values are presented as mean \pm standard error of mean (SEM). Statistical significance was determined using two-tailed Student's t-test for comparison between two groups or two-way ANOVA with Holm-Sidak's multiple comparisons test for comparison between multiple groups. Statistical significance was set at $\mathrm{P}<0.05$. 


\section{ACKNOWLEDGEMENTS}

This work was supported by the National Research Foundation of Korea (NRF) grant funded by the Korea government (MSIP) (No. 2017R1A2B3004565).

\section{CONFLICTS OF INTEREST}

The authors have no conflicting interests.

\section{REFERENCES}

1. Shibuya M (2011) Vascular endothelial growth factor (VEGF) and its receptor (VEGFR) signaling in angiogenesis: a crucial target for anti- and pro-angiogenic therapies. Genes Cancer 2, 1097-1105

2. Beck B, Driessens G, Goossens $S$ et al (2011) A vascular niche and a VEGF-Nrp1 loop regulate the initiation and stemness of skin tumours. Nature 478, 399-403

3. Kim JY and Kim YM (2019) Tumor endothelial cells as a potential target of metronomic chemotherapy. Arch Pharm Res 42, 1-13

4. Kerbel RS (2008) Tumor angiogenesis. N Engl J Med 358, 2039-2049

5. Stoelting S, Trefzer T, Kisro J, Steinke A, Wagner T and Peters SO (2008) Low-dose oral metronomic chemotherapy prevents mobilization of endothelial progenitor cells into the blood of cancer patients. In Vivo 22, 831-836

6. Kerbel RS and Kamen BA (2004) The anti-angiogenic basis of metronomic chemotherapy. Nat Rev Cancer 4, 423-436

7. Räsänen M, Degerman J, Nissinen TA et al (2016) VEGF-B gene therapy inhibits doxorubicin-induced cardiotoxicity by endothelial protection. Proc Natl Acad Sci U S A 113, 13144-13149

8. Park M, Kim J, Kim T et al (2021) REDD1 is a determinant of low-dose metronomic doxorubicin-elicited endothelial cell dysfunction through downregulation of VEGFR-2/3. Exp Mol Med (In press)

9. DeYoung MP, Horak P, Sofer A, Sgroi D and Ellisen LW (2008) Hypoxia regulates TSC1/2-mTOR signaling and tumor suppression through REDD1-mediated 14-3-3 shuttling. Genes Dev 22, 239-251

10. Lee DK, Kim JH, Kim J et al (2018) REDD-1 aggravates endotoxin-induced inflammation via atypical NF- $\mathrm{KB}$ activation. FASEB J 32, 4585-4599

11. Karar J and Maity A (2011) PI3K/AKT/mTOR Pathway in Angiogenesis. Front Mol Neurosci 4, 51

12. Guba M, von Breitenbuch P, Steinbauer $M$ et al (2002) Rapamycin inhibits primary and metastatic tumor growth by antiangiogenesis: involvement of vascular endothelial growth factor. Nat Med 8, 128-135
13. Wang M, Xu Y, Wen GZ, Wang Q and Yuan SM (2019) Rapamycin suppresses angiogenesis and lymphangiogenesis in melanoma by downregulating VEGF-AVVEGFR-2 and VEGF-CNEGFR-3 expression. Onco Targets Ther 12, 46434654

14. Marumo $T$, Uchimura $H$, Hayashi $M$, Hishikawa $K$ and Fujita T (2006) Aldosterone impairs bone marrow-derived progenitor cell formation. Hypertension 48, 490-496

15. Pitchford SC, Furze RC, Jones CP, Wengner AM and Rankin SM (2009) Differential mobilization of subsets of progenitor cells from the bone marrow. Cell Stem Cell 4, 62-72

16. Aicher A, Heeschen C, Mildner-Rihm C et al (2003) Essential role of endothelial nitric oxide synthase for mobilization of stem and progenitor cells. Nat Med 9, 1370-1376

17. Bertolini F, Paul S, Mancuso P, Monestiroli S, Gobbi A Shaked Y and Kerbel RS (2003) Maximum tolerable dose and low-dose metronomic chemotherapy have opposite effects on the mobilization and viability of circulating endothelial progenitor cells. Cancer Res 63, 4342-4346

18. Bocci G, Francia G, Man S, Lawler J and Kerbel RS (2003) Thrombospondin 1, a mediator of the antiangiogenic effects of low-dose metronomic chemotherapy. Proc Natl Acad Sci U S A 100, 12917-12922

19. Saif MW (2013) Anti-VEGF agents in metastatic colorectal cancer (mCRC): are they all alike? Cancer Manag Res 5, 103-115

20. Asahara T, Murohara T, Sullivan A et al (1997) Isolation of putative progenitor endothelial cells for angiogenesis. Science 275, 964-967

21. Asahara T, Masuda H, Takahashi T et al (1999) Bone marrow origin of endothelial progenitor cells responsible for postnatal vasculogenesis in physiological and pathological neovascularization. Circ Res 85, 221-228

22. Willett CG, Boucher Y, di Tomaso E et al (2004) Direct evidence that the VEGF-specific antibody bevacizumab has antivascular effects in human rectal cancer. Nat Med 10, 145-147

23. Murakami J, Li TS, Ueda K, Tanaka T and Hamano K (2009) Inhibition of accelerated tumor growth by blocking the recruitment of mobilized endothelial progenitor cells after chemotherapy. Int J Cancer 12, 1685-1692

24. Sxton RA and Sabatini DM (2017) mTOR Signaling in growth, metabolism, and disease. Cell 169, 361-371

25. Giraud S, Greco A, Brink M, Diaz JJ and Delafontaine P (2001) Translation initiation of the insulin-like growth factor I receptor mRNA is mediated by an internal ribosome entry site. J Biol Chem 276, 5668-5675

26. Chen TG, Chen JZ and Wang XX (2006) Effects of rapamycin on number activity and eNOS of endothelial progenitor cells from peripheral blood. Cell Prolif 39, $117-125$ 